\title{
Design of Power Grid Intelligent Patrol Operation and Maintenance System Based on Multi-Rotor UAV Systems
}

\author{
Qunying Liu ${ }^{\mathrm{a}, 1}$, Haifeng Zenga ${ }^{\mathrm{a}}$, Shaojian $\mathrm{Ni}^{\mathrm{a}}$, Bowen $\mathrm{Li}^{\mathrm{a}}$, Jingsong Meng ${ }^{\mathrm{a}}$, and Yiguo \\ Zhang ${ }^{\mathrm{b}}$ \\ ${ }^{a}$ School of Automation and Engineering, University of Electronic Science and \\ Technology of China \\ ${ }^{\mathrm{b}}$ Guangzhou Polytechnic of Science and Trade
}

\begin{abstract}
Complex geographical conditions and bad weather have caused great difficults to inspect transmission line of power grids. This work aims to develop a micro multi-rotor Unmanned Aerial Vehicle (UAV) system for inspecting power grid. The proposed system integrates with an intelligent robot and mobile communication networks. The high-resolution aerial images of the inspection line can be obtained to improve the operational efficiency and safety. The time period from the front-end signal acquisition to terminal decoding and playback is $1.5 \mathrm{~s}$ for condition-based maintenance. The system supports TDD-LTE, FDD-LTE, WCDMA, CDMA2000, and other network standards. It also supports the video input signals with the resolution of standard 576i, HD 720p, FHD 1080i, and FHD $1080 \mathrm{p}$. It is compatible with a variety of transmission formats and codes, help to achieve timely, comprehensive and efficient high-resolution aerial image acquisition of patrol lines, and improve operational efficiency and safety, real-time monitoring and condition-based maintenance of power transmission lines.
\end{abstract}

Keywords: Multi-Rotor UAV System, Intelligent Patrol Operation, Path Planning, Power Grid Maintenance

\section{Introduction}

The fault detection technology developed for the power grid includes the methods based on impedance, methods based on the traveling wave, and methods based on sample training [1]. Impedance-based methods [2] have been applied to determine the fault position typically by using voltage and current measurements from one or more terminals to calculate line impedance. The impedance-based method, especially for the one using local-measurements, is only based on reactance by ignoring the influence of fault resistance and load current. However, this method may produce significant errors caused by remote end current infeed, load impedance, power transmission angle and angle difference between the transmission line and electricity source impedances [3]. To meet the increasing demand for electricity and the safe operation of grid, automation and modernization of line patrol maintenance have increasingly shown their urgency[4]. With the development of Unmanned Aerial Vehicle (UAV) technology [5], the

\footnotetext{
${ }^{1}$ Corresponding Author, Qunying Liu, School of Automation Engineering, University of Electronic Science and Technology of China, Chengdu 611731, China; E-mail: lqy1206@126.com.
} 
application of UAV in power grid patrol has entered an exploratory stage, it is becoming an important method for power grid maintenance. An automatic electrical equipment abnormal heat diagnosis method using infrared image sequence adopted from Unmanned Helicopter Vehicle (UHV) is proposed in [6], which automatically identifies the locations of device fault for further manual inspection. The traditional mode of operation and inspection has the following problems [7]: (1) information acquisition mode is traditional and single source; (2) equipment condition perception is restricted by power outage maintenance and off-line experiments; (3) Limited usage of advanced data utilization tools such as online monitoring, live maintenance. Under these conditions, the concept of intelligent transportation and inspection based on the modern information technology of "Big Cloud Moving" emerged as the times require [8]. UAV-assisted power grid patrol is an essential part of the three-dimensional patrol system based on Intelligent equipment. Based on the application status and possible development direction of UAV-assisted power grid patrol operation[9], this paper develops a system, namely the Power Grid Intelligent Patrol UAV System (Esmart), which significantly boosts the level of automation, intelligence and efficiency of the power grid patrol using latest computer mobile communication technology. The power grid intelligent inspection UAV system is a vital part of the three-dimensional inspection system based on the intelligent equipment and the current inspection technology research. The UAV-assisted power grid patrol can effectively solve the problems of traditional manual patrol, such as large workload, low efficiency, high risk, high cost of manned helicopter patrol, complex flight approval procedures, and limited operating environment. With the development of application technology in the UAV industry [10,11,12], UAV-assisted power grid patrol will provide a safe, efficient and comprehensive patrol mode for power grid patrol to meet the needs of daily power grid patrol, fault patrol, and emergency rescue.

This work aims to develop a micro multi-rotor UAV system for power grid inspection. The proposed system adapts to various patrol scenarios by combining the key technologies in the intelligent robot, mobile communication, and geographic information. The intelligent patrol UAV system and software for the power grid, namely Esmart, is developed independently based on the iOS mobile operating system. Automated intelligent control software system for deep customization of Power Patrol operation. The main functions of the system include: KML, Excel files import, flexible flight parameter setting, automatic route planning, one-button takeoff and landing, automatic operation, intelligent safety inspection, real-time image transmission. Panoramic acquisition module, refined patrol module, channel/tree barrier patrol module, and rapid mapping and other intelligent business operation modes can meet the data acquisition needs of various application scenarios, can timely, comprehensive and efficient access to high-resolution aerial photographs of patrol lines, improve operational efficiency and ensure operational safety, real-time monitoring and condition-based maintenance of power transmission lines. 


\section{Micro multirotor multispectral UAV system equipment (UAV carrier multi- sensor integration)}

\subsection{The system specifications}

The micro multi-rotor multi-spectral UAV system equipment is mainly composed of a micro multi-rotor UAV and a multi-spectral camera. The multi-spectral camera is used for acquisition band range including $(450-520 \mathrm{~nm}),(520-600 \mathrm{~nm}),(630-690 \mathrm{~nm}),(760-$ $900 \mathrm{~nm}$ ) and RGB. Therefore, the processing and analysis of the multi-spectral and multiband images rely on professional software, which provides a scientific basis for water quality by detecting and analyzing the spectral information in different environments as well as engineering monitoring.

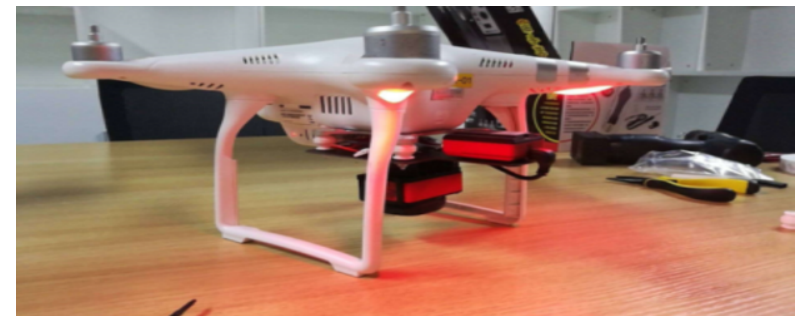

Figure 1. Micro multirotor UAV system equipment

The micro multirotor UAV system equipment is shown in Figure 1. The detail parameters of the UAV system are shown in the following. The product positioning: professional film and television and commercial aerial photography machine weight: 1380g; Hovering accuracy: vertical: /-0.1m (ultrasonic working range); /-0.5m level:/$0.3 \mathrm{~m}$ (within the working range of visual sensor): $/ 1.5 \mathrm{~m}$. Lift speed: maximum rise speed: $6 \mathrm{~m} / \mathrm{s}$ (motion mode) ,maximum drop speed: $4 \mathrm{~m} / \mathrm{s}$ (motion mode). flight speed 20m/s (motion mode). flight altitude: $6000 \mathrm{~m}$. Flight time about 28 minutes. Wheelbase $350 \mathrm{~mm}$.Propeller 9450S quick-release paddle. Tele-controller: Operating frequency band: 2.4-2.5GHZ. Operating voltage:7.5v. Receiving gain: $10 \mathrm{~dB} \pm 1$. Emission gain: $15 \mathrm{~dB} \pm 1$. The input power range: $3 \sim 20 \mathrm{dBm}$. The maximum output power is $4 \mathrm{~W}(36 \mathrm{dBm})$. Operating current: 435Ma@Pout28dBm9V; Noise coefficient $\leq 2.5 \mathrm{db}$. Transmission delay $\leq 1$ us. The operating temperature: $40 \sim 80^{\circ} \mathrm{C}$. The control distance: $5,000 \mathrm{~m}$ (No interference, no shielding); CE: $3500 \mathrm{~mm}$.

In this system, a Multi-spectral sensor is embedded. A 2 mega-pixel monochromatic image sensor collects data on the discrete spectral band: green light $(550 \mathrm{~nm}$, bandwidth $40 \mathrm{~nm})$, red light $(660 \mathrm{~nm}$, bandwidth $40 \mathrm{~nm})$, red edge light $(735 \mathrm{~nm}$, bandwidth $10 \mathrm{~nm})$, and near-infrared light $(790 \mathrm{~nm}$, bandwidth $40 \mathrm{~nm})$. The supported image sensor settings include green light, red-edge light, near-infrared light, RGB and so on. In addition, the system contains a 16 mega-pixel RGB trichromatic image sensor. It also includes a magnetometer, a Wi-Fi wireless network signal interface. The micro USB host port connects multispectral sensor to the sunshine sensor. The Micro USB port equipment connects multispectral sensors and unmanned drivers. The other components of the multispectral sensor are used as the inertial navigator. 


\section{System Functions}

Esmart, which responsible for data acquisition of the power grid intelligent patrol UAV system as shown in Figure 2. Esmart is a flight control software of intelligent aerial survey UAV developed based on the iOS mobile operating system and Xinjiang SDK. It integrates functions of automatic cruise, route planning and auxiliary control. It also supports common aircraft types in Xinjiang and capture power line images as well as environment information, which are important for decision making and understanding failure mechanism in long-term studies.

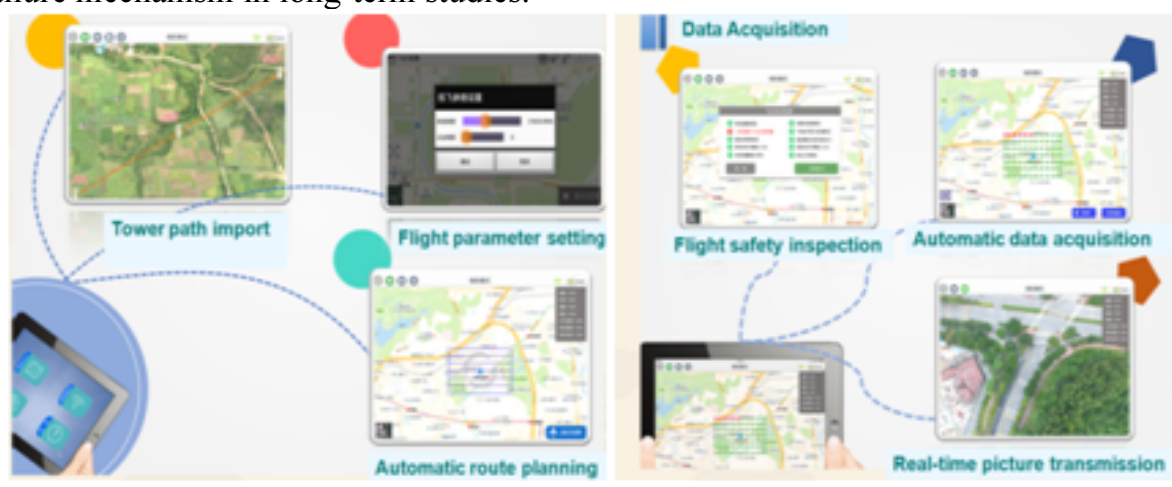

Figure 2. Functional architecture of intelligent patrol UAV system in the power grid

Esmart is customized in-depth according to the characteristics of Power Patrol operation. It has KML, excel file import, flexible flight parameter setting, automatic route planning, one key takeoff and landing, automatic operation, intelligent safety inspection, real-time image transmission and other general functions. Panoramic acquisition module, fine inspection module, channel / tree barrier inspection module and rapid mapping and other intelligent business operation modes can meet the data collection requirements of various application scenarios, and can obtain high-resolution aerial photos of inspection lines in a timely, comprehensive and efficient manner, by which the Esmart can improve the operation efficiency and ensure the operation safety of UAV system. It can be further developed for Power Grid Intelligent Patrol Operation and Maintenance System.

It develops the following modules to satisfy various data acquisition of transmission/distribution lines in power industry: (1) Panoramic acquisition module, which is used for aerial photography acquisition of 360-degree panoramic images, mainly used for channel inspection of transmission and distribution lines and substation inspection, and can quickly acquire 360-degree panoramic images on site; (2) Fine patrol module, which is used for fine photographic inspection of key equipment's and key parts on poles and towers, and image collection of every potential defective part to provide data support for later intelligent classification and defect analysis; (3) Channel/tree barrier patrol module, which is used for Fully automatic patrol of corridors and corridors of lines can be realized by combining images in the later stage, which can provide reliable basis and guarantee for avoiding potential safety hazards caused by tree barrier problems; (4) Rapid mapping, which can be used to collect orthophoto and oblique photographic data of platform and sheet areas, can be realized in the later stage. Three-dimensional modeling can be widely used in planning, infrastructure, maintenance, monitoring and other processes in the power industry; (5) coordinate management, which is used to 
update the coordinates of line poles and towers, facilitates the daily maintenance of basic data of power equipment, and improves the accuracy of internal network system data.

\subsection{The Intelligent Control system of UAV Inspection (automatic Inspection path Planning of $U A V)$}

To improve the efficiency of UAV inspection and realize the standardization of UAV inspection, an automatic patrol path planning technology of UAV is developed in this work. The proposed method plans a patrol route according to the positions of power grid towers, the status of the signal channel, environmental barriers, orthophoto image acquisition and tilt photography. For the route planning of orthography and tilt photography in fast mapping, path is calculated by using the inverse triangle tangent method [13] and the position of touch point on the screen of the mobile device. The multiple parameters of KML, images and videos from panoramic acquisition will be processed and monitored through feature extraction and information mining for health monitoring with spatial geographic information data, abnormal diagnostics and further prevention including condition-based maintenance.

Specifically, the patrol route of the channel or tree barrier is automatically planned by calculating the route inspected by the UAV based on the imported line file tower coordinates, the overlap parameters of the flight beat, and the parameters of the aircraft flight. Fast mapping (orthophoto and tilt photography) selects the mapping area on the map through manual interaction, then the route inspected by UAV can be automatically calculated according to the overlap parameters of aerial photography and the parameters of aircraft flight. After the route is calculated, the multi-rotor UAV enters to the autonomous flight mode. For the failed UAV, such as the whom have completed their mission or lost signal connection with the controller, will return to the departure point automatically. In addition, when the operation area is too large to allow the UAV to complete the required mission, the UAV can divide the patrol route into different sections automatically according to the battery level. Each section contains a break point to allow the UAV to land and replace the battery.

In addition, each module of the system contains mission management function, which records the mission of each flight, including the parameters of flight setting, and flight path, supports task viewing and can be loaded and executed again. In this way, the standardization of inspection operation can be guaranteed, and the efficiency of inspection is improved as well. The inspection situation of UAV can be transmitted back in real-time through satellite transmission and local wireless communication. The field situation can be displayed in controller intuitively, which provides reference for command and decision making. Ground station monitoring and remote image transmission system are compatible with the patrol multi-rotor UAV system, micro multi-rotor multi-spectrum UAV system, as well as the power patrol inspection and water conservancy patrol remote monitoring system to provide remote emergency monitoring service. The system platform supports the real-time and stable transmission of the pictures captured by the multi-rotor UAV in the sky to the ground wireless image transmission and remote-control receiving equipment. The video transmitter receives digital or analog signals from the UAV, then generates and transmits audio and video signals to the monitoring center through various network modes such as EDGE, EVDO, TD-SDCDMA, WCDMA and LTE, then broadcast the multi-media data to various platforms (TV, smart phones, and etc.) through the server for display. The whole work process of the system is shown in Figure 3. 


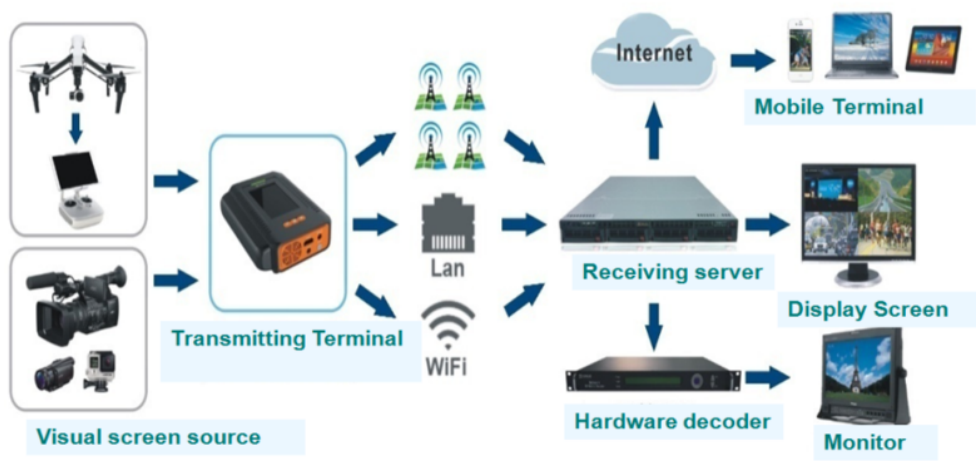

Figure 3. The work process of system

The image remote transmission terminal is based on multi-link aggregation technology and H.265 coding technology real-time video transmission terminal. Equipped with three built-in $4 \mathrm{G}$ modules and a USB extension interface, while supporting Wi-Fi and wired network access. The image transmission enhancement terminal is a bidirectional power amplifier developed for $2.4 \mathrm{GHz}$ band and in accordance with IEEE $802.11 \mathrm{~b} / \mathrm{g} / \mathrm{n}$ standard. The product matches the frequency expansion techniques such as direct sequence (DSSS), orthogonal frequency division reuse (OFDM). Time Division Duplex (TDD) fast microwave detection technology and linear power amplifier technology are used to greatly expand the radio frequency communication distance under the condition that the transmission rate of $802.11 \mathrm{~b} / \mathrm{g} / \mathrm{n}$ wireless equipment remains unchanged.

\subsection{Intelligent Flight}

The UAV has a set of intelligent operation functions, including automatic operation mode, one-button take-off, self-operation, automatic return. The workflow of the onebutton automatic takeoff is as follows: set the flight mode and parameters, start the task, the software starts to automatically check the flight parameters, as shown in Figures 4 and 5, after completing the pre-flight safety check, slide key, one-button take-off of the aircraft, self-operation, automatic execution of flight tasks. The workflow of the fullautomatic operation mode is as follows: click the automatic take-off key after completing the pre-flight safety check. When the flight altitude reaches $2 / 3$ of the flight altitude of the first break point, the aircraft automatically adjusts the direction of the platform to the preset angle of the platform. After arriving at the first breakpoint, it carries out automatic flight and execution tasks according to the preset route (e.g. taking photos).

Flight status information is transmitted to controller in real time to allow the operator to check the flight status of the UAV. In case of emergency (such as signal lost or insufficient energy), one-click return, or suspension can be made to allow the aircraft return to the last take-off point or hover in the position temporarily. 


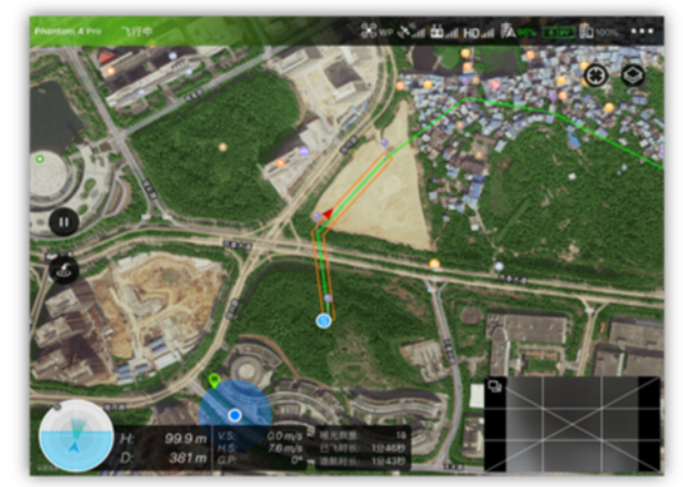

Figure 4. Take off to carry out a mission

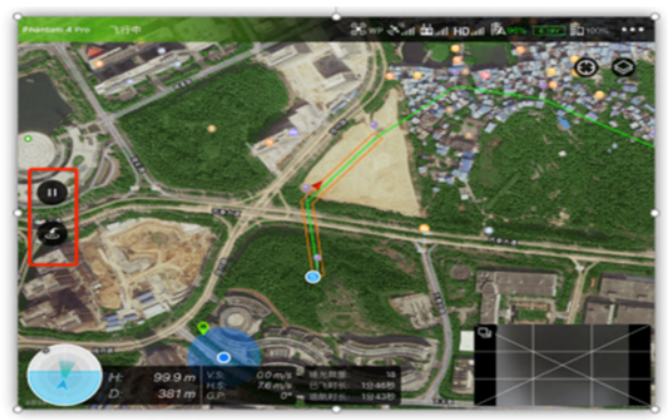

Figure 5. Suspend flight or return by one click

\section{Conclusion}

The inspection for power grid has always been a challenging task, because of the complex structure of electrical equipment and the physical distribution characteristics of the power grid. In addition, the long monitoring distance, harsh geographical and climatic conditions bring huge difficulties to manual inspection. To achieve low-cost high efficiency inspection of power grid, this paper presents the development of an intelligent UAV based power grid inspection system, which improves the standardized line management and emergency command. The rapid and intelligent collection processing and application of spatial geographic information data in the key areas of power grid line corridor in collaboration with failure diagnostics and health management are realized, by which the needs of fine grid inspection, channel inspection, fault inspection and special inspection can be met. One of the premises of this conclusion is that the control inputs aren't delayed. However, according to the experiment results, there is often a delay in the actual communication, and the force and torque output by the actuator such as the motor are delayed. Moreover, the response may not keep up with the change of the control amount, so the forces acting on the aircraft are delayed, which may cause the response of the flight system to be slow or even unstable. Now the problem is that when a disaster occurs at early warning, it is too easy to be impacted to implement the command of disaster monitoring and auxiliary disaster relief, providing timely, intuitive, and comprehensive auxiliary decision-making basis for disaster control. It has 
practical and efficient advantages for power grid inspection and has the prospect of promotion and application. As a result, it is recommended to promote the application in a wider range of industries.

Acknowledgments: This work in this paper is supported by the Sichuan Province Science and Technology supporting plan (No.2019YFG0129).

\section{References}

[1] M. Majidi and M. Etezadi-Amoli, A new fault location technique in smart distribution networks using synchronized/nonsynchronized measurements, IEEETransactionsonPowerDelivery,2018,33(3):13581368.

[2] Tirath Pal S. Bains, Tarlochan S. Sidhu, Zhihan Xu, Ilia Voloh and Mohammad R. Dadash Zadeh, Impedance-Based Fault Location Algorithm for Ground Faults in Series-Capacitor-Compensated Transmission Lines, IEEE Transactions on Power Delivery, 2018,33(1):189-199.

[3] M. Kezunovic, Smart Fault Location for Smart Grids," IEEE Transactions on Smart Grid, vol.2, no.1, pp.11-22,2011.

[4] Li Bingqiang, Wang Qian. Applying unmanned autonomous helicopter to transmission line inspection[J]. Shandong Electric Power, 2010, 171 (1):1-4(in Chinese)

[5] Chenhao Zhang, Guobing Song, Ting Wang and Liming Yang, SingleEnded Traveling Wave Fault Location Method in DC Transmission Line Based on Wave Front Information, IEEE Transactions on Power Delivery,2019,34(5):2028-2238.

[6] Zhang Wenfeng, Peng Xiangyang, Chen Ruimin, Cnen Chi, Deng Chaoyi, QIAN Jinju, XU Wenxue. Intelligent Diagnostic Techniques of Abnormal Heat Defect in Transmission Lines Based on Unmanned Helicopter Infrared Video[J]. Power System Technology,2014,38(5):1334-1338

[7] Jinju Qian, Xiaoming Ma, Ke Wang, Lin Yi, Xiaoyang Peng, Zhangquan Rao. Application and effect of large-scale Inspection Power Lines by Using Large Unmanned Helicoper in Guangdong Power Grid. Guangdong Electric Power, 2016,29(5):124-129.

[8] Zhonghua Hu, Min Zhao, Min Yao. Research and Development Trend of Path Planning for Unmanned Air Vehicle[J]. Avionics Technology, 2009, 40(2):24-29.

[9] Xiangyang Peng, Qing Zhong, Zhangquan Rao. Intelligent Diagnostic Techniques of Transmission Lines Corona Dischare Defect based on Ultraviolet Detection from Unmanned Aerial Vehicle. High Voltage Engineering,2014,40(8):2292-2298.

[10] Jianchao Li, Liming Wang, Xinwei Shen. Unmanned Aerial Vehicle Intelligent PatrolInspection System Applied to Transmission Grid.2018 IEEE Conference on Energy Internet and Energy System Integration (EI2),pp.1-6.

[11] Homma, R. Z., Sohn, O., \& Bose, R. C. Analysis of the recognition and localisation techniques of power transmission lines components in aerial images acquired by drones. CIRED-Open Access Proceedings Journal, 2017(1), 29-32.

[12] Zhuofan Xu, R. Wei, Qirui Zhang, Kai Zhou and Renke He, Obstacle avoidance algorithm for UAVs in unknown environmentbased on distributional perception and decision making, 2016 IEEE Chinese Guidance, Navigation and Control Conference (CGNCC), Nanjing, 2016, pp. 1072-1075.

[13] H. Kandath, T. Bera, R.Bardhan and S. Sundaram. Autonomous Navigation and Sensorless Obstacle Avoidance for UGV with Environment Information from UAV, 2018 Second IEEE International Conference on Robotic Computing (IRC), Laguna Hills, CA, 2018, pp. 266-269. 